\title{
YALE OBSERVATION SCALE AS A PREDICTOR OF BACTEREMIA AND FINAL OUTCOME IN 3-36 MONTHS OLD FEBRILE CHILDREN ADMITTED IN TERTIARY HEALTH CENTRES: A HOSPITAL-BASED CROSS-SECTIONAL STUDY
}

\author{
SHAGUN WALIA, HARIS MM, ALI KUMBLE, SOUNDARYA M*, BALIGA BS, CHETAN KUMAR N
}

Department of Paediatrics, Kasturba Medical College, Mangalore, Karnataka, India. Email: soundarya29@gmail.com Received: 15 March 2016, Revised and Accepted: 05 September 2016

\section{ABSTRACT}

Objectives: The objective of the study was to assess predictability of bacteremia in febrile children in the age group of 3-36 months by application of Yale observation scale (YOS) and to predict clinical course during hospital stay and final outcome by YOS.

Methods: A hospital-based cross-sectional study was carried out at Kasturba Medical College, Mangalore, Karnataka, for a period of 2 years (September 2013-September, 2015) in 100 febrile children in the age group of 3-36 months with probable infectious etiology admitted in ward/PICU. Children with any non-infectious causes of fever (vaccination, autoimmune, and immunodeficiency disorder) were excluded from the study. Cases were selected by simple random sampling. The primary study outcome was bacteremia based on positivity on blood culture and sensitivity sample drawn at admission. Secondary outcomes are clinical course in the hospital, use of antibiotics, need for mechanical ventilation, hospital stay, and mortality.

Results: 100 cases were included in the study out of which 18 cases were bacteremic with a mean YOS of 26 (non-bacteremic - 11), mean hospital stay 19.5 days (non-bacteremic - 12 days). All 18 bacteremic children had YOS $\geq 20$, but YOS $\geq 20$ had 8 false positives cases. There was no significant interobserver variability in YOS assessment (Cronbach's alpha - 0.993 showing good correlation with intraclass correlation coefficient - 0.986). Higher YOS scores had good sensitivity, specificity, positive and negative likelihood ratios, and area under curve for prediction of bacteremia at YOS $>20(100 \%, 90.2 \%, 10.2,0.00$, and 0.970$)$, need for mechanical ventilation at YOS $>21(100 \%, 91.7 \%, 12.04,0.00$, and 0.969$)$, need for scaling up antibiotics at YOS >21 (70.4\%, 94.4\%, 12.5, 0.31, and 0.822), and mortality at YOS > 21 (90.9\%, 85.4\%, 6.2, 0.106, 0.878).

Conclusion: YOS is a good tool to rule out bacteremia and to prognosticate the clinical course at the first visit. This simple scale can be of value in monitoring admitted patients for deteriorating clinical state and for assessing the need for referral to higher centers for further management.

Keywords: Yale observation scale, Bacteremia, Febrile patients.

(C) 2016 The Authors. Published by Innovare Academic Sciences Pvt Ltd. This is an open access article under the CC BY license (http://creativecommons. org/licenses/by/4. 0/) DOI: http://dx.doi.org/10.22159/ajpcr.2016.v9s3.11707

\section{INTRODUCTION}

Sepsis is one of the leading causes of mortality in children under 5 years age by UNICEF statistics [1]. Occult bacteremia presents initially with non-specific non-localizing symptoms resulting in delayed recognition and treatment and mortality thereafter. Laboratory investigations can only be used as supporting evidences with blood culture and sensitivity being the gold standard to confirm bacteremia (results take 48-72 hrs) leaving clinical assessment as the major tool for early diagnosis.

One such clinical tool is Yale observation scale (YOS) which is a simple, quick, easy to apply, and cost-effective [2] bed-side scale clubbed in 1982 by McCarthy et al. [3] which has 6 parameters validated in young febrile children for gradation of toxicity of the clinical state. Majority of the previous studies on YOS focus on the prediction of serious bacterial illnesses (SBIs) in febrile children. Very few studies have been reported on the role of YOS in the prediction of bacteremia.

We assessed the ability of YOS in predicting bacteremia and to prognosticate a child's clinical course at admission.

\section{METHODS}

A hospital-based cross-sectional study was carried out in Kasturba medical college, Mangalore, from September 2013-September 2015 after taking approval from the Institutional Ethics Committee. Cases were selected by simple random sampling.
With the prediction of 0.5 , the estimated sample size using the following formula is $75, \mathrm{n}=2(\mathrm{Z} \alpha+\mathrm{ZB})^{2} / \mathrm{C}^{2}+3=75$ [where $\mathrm{C}=0.5 * \ln$ $(1+r / 1-r), r=$ correlation coefficient, $Z \alpha=1.96$ (95\% confidence level), $\mathrm{Z} \mathrm{b}=1.28$ (90\% power)].

100, 3-36 months old, febrile infants and children were assessed after taking prior informed consent from the parents (an early morning temperature of $98.5 \mathrm{~F}$ or higher temperature of $99.4 \mathrm{~F}$ or higher at any time during the day was defined as fever). The cases with any noninfectious causes of fever such as vaccination in the past 2 days, suspicion of any immunodeficiency, or autoimmune disorder were excluded from the study. Axillary temperature of the selected cases was documented, following which their YOS was assessed by two independent observers ranging on a scale of 6-30 ( 6 being the healthy children and 30 being the sickest ones) YOS takes into consideration 6 parameters including quality of cry, state of hydration, color, state of wakefulness, response to parental stimulation, and to social overtures such as playing, talking, and smiling. The parameters were graded for normal, moderate, or severe impairment with 1, 3, and 5 scores. After YOS assessment, detailed evaluation (history and physical examination) of the child was done. Blood samples were sent for culture and sensitivity and other relevant investigations. The data were entered into a well-structured pro forma. During the time of hospital stay, the child was treated by consultants who were blinded to YOS. Details of further management including antibiotics usage, requirement of mechanical ventilation, and final diagnosis were obtained from the case sheets when they were discharged or following death. 
Outcome parameters were:

A. Primary outcome:

- Bacteremia

B. Secondary outcomes:

- Requirement of mechanical ventilation

- Number of times scaling up of antibiotics was done

- Final outcome - recovered/expired.

The collected data were analyzed using ROC curve for finding cut-off scores of YOS for prediction of bacteremia, requirement of ventilation support, final outcome, and the number of times antibiotics were changed with the calculation of the respective sensitivities, specificities, and likelihood ratios. Interobserver variability for the YOS was assessed using Cronbach's alpha and intraclass correlation coefficient.

SPSS 17.0 software was used for the above analysis.

\section{RESULTS}

100 cases were included in the study out of which 18 cases were bacteremic with a mean YOS of 26 (non-bacteremic - 11), mean hospital stay 19.5 days (non-bacteremic - 12 days). ROC curves and statistical analysis for the prediction of bacteremia and clinical outcome are mentioned in Tables 1-4. Table 4 shows the effect of increasing YOS scores on the outcome parameters.

The analysis for interobserver reliability of YOS was assessed using Cronbach's alpha which was 0.993 showing good correlation with intraclass correlation coefficient being 0.986 .

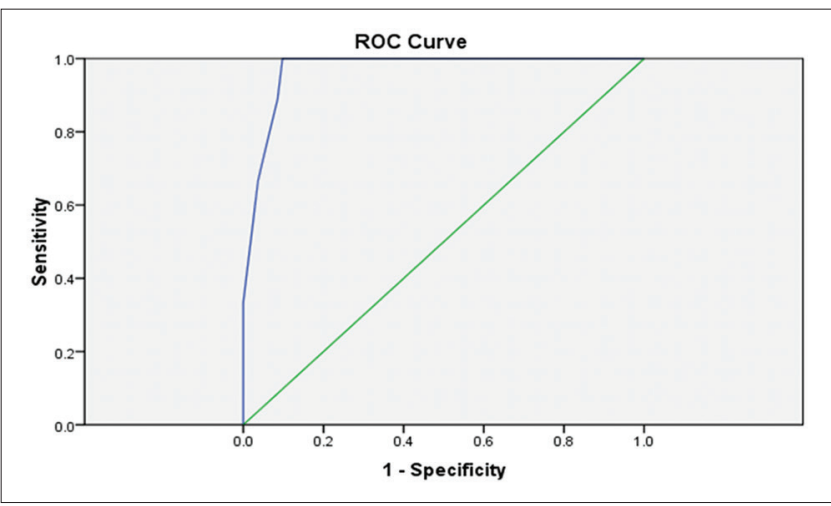

Fig. 1: Receiver operating characteristics curve for prediction of bacteremia by Yale observation scale

Table 1: Reciever operating characteristics curve for prediction of bacteremia by YOS

\begin{tabular}{|c|c|c|c|}
\hline \multicolumn{4}{|c|}{ Test result variable: YOS scale } \\
\hline \multicolumn{3}{|l|}{ Coordinates of the curve } & \multirow{2}{*}{$\frac{\text { Bacteremia }}{\text { Specificity }}$} \\
\hline $\begin{array}{l}\text { Positive if YOS greater } \\
\text { than or equal To }{ }^{\mathrm{a}}\end{array}$ & Sensitivity & 1-specificity & \\
\hline 19.00 & 1.000 & 0.098 & 0.902 \\
\hline 21.00 & 0.889 & 0.085 & 0.915 \\
\hline
\end{tabular}

YOS: Yale observation scale

Table 2: Prediction of bacteremia by YOS

\begin{tabular}{lll}
\hline YOS score $^{* *}$ & Bacteremia & Others \\
\hline$>0 \mathrm{r}=20$ & 18 & 8 \\
$<20$ & 0 & 74 \\
\hline
\end{tabular}

**Sensitivity - 100\%, specificity - 90.2\%, PPV: Positive predictive value - $69 \%$, NPV: Negative predictive value - $100 \%$, positive LR - 10.2, negative LR - 0.00 , YOS: Yale observation scale

\section{DISCUSSION}

In the McCarthy et al. study,1982 [3], YOS was made from 6 clinical parameters to grade the toxicity of the clinical state of a child. The sensitivity of YOS to detect serious bacterial infections was $92.3 \%$ with a score $>16$ in the above study. Subsequently, the scale has been assessed in 7 studies [5,12]. The YOS had little value in confirming (positive likelihood ratio range 1.10-6.70) or excluding (negative likelihood ratio range $0.16-0.97)$ the possibility of serious infection. It provided a LR $\leq 0.2$ in McCarthy et al. and Thayyil et al. [3,6] (Table 5), whereas in five other studies, it varied from 0.68 to 0.94 [7-11]. The summary sensitivity of these five studies was 32.5\% (95\% CI: 21.7 $45.5 \%$ ) and specificity was $78.9 \%$ (95\% CI: 73.9-83.1\%), theoretically corresponding to a $\mathrm{LR}+$ of 1.54 and a $\mathrm{LR}-$ of 0.86 .

Table 5 shows various studies carried out on YOS. First, statistical value of a test changes with prevalence of disease in the study population. For instance, the prevalence of bacteremia and SBIs in our study was $18 \%$ and $44 \%$ as against Hsiao et al. where it was $0.9 \%$ and $10.3 \%$ [10], respectively. Second, high-risk infants with more probability of bacteremia such as toxic children and fever without focus were included in above studies (fever was the only clinical criteria in our study). Third, the best likelihood ratios in the above studies were of McCarthy et al. (1982) probably due to low vaccine coverage rates (in 1982, vaccine coverage rates for the USA for DTP 3 dose were only $67.1 \%$ as against $94.3 \%$ in 2012 [13]; DTP 3 coverage by 12 months of age is a key indicator of immunization program performance [14]) and hence higher prevalence of the disease. The same may be the reason for good performance of the YOS in our setting (DTP 3 coverage in India was only $72 \%$ in 2013 [15] contributing to the maximum number of DTP 3 unimmunized children globally [14]).

Age of the study population is very important for the assessment of YOS as it depends on neurological maturity of the child, hence for studies to be comparable, the age of children should be constant. In view of inconstancy in the selection parameters regarding the prevalence of disease and age, the results of the above studies cannot be extrapolated to all children and settings.

Although YOS scores of $\geq 20$ predict bacteremia, it is not a good scale to rule in bacteremia as the specificity is low (Tables 1 and 2). However, it is a good scale to rule out bacteremia as NPV and sensitivity of the scale are $100 \%$. Therefore, YOS is a good clinical assessment tool to initiate empirical antibiotics therapy till bacteremia is proven by blood culture growth, if YOS of a child is $\geq 20$. This has important implications as early, and appropriate antibiotics improve outcome in bacteremia. Other factors responsible for poor YOS scores were identified including acute kidney injury, post-ictal state, H1N1 infection, and severe pneumonia with impending respiratory failure constituting the false positives (bacteremia being true positive) for YOS $>20$.

YOS $>21$ has prognostic (increased requirement of mechanical ventilation, mortality, and need to scale up antibiotics) implications (Table 3) irrespective of etiology. Hence, we conclude that YOS >21 in a febrile child of age 3-36 months signifies toxic state requiring aggressive management based on the clinical presentation.

The analyzed cases were organized into subgroups of increasing YOS scores from 6 to 30 (4 subgroups) as in Table 4 against the abovementioned clinical outcome parameters. Table 4 shows that as the YOS score increases, the clustering of cases changes from good clinical outcome (no requirement of mechanical ventilation, recovery with treatment, and no need for scaling up antibiotics) to poor clinical outcomes at higher scores, hence signifying the applicability of YOS to monitor admitted patients for diagnosing refractoriness to ongoing treatment in advance, but this hypothesis needs validation by further studies.

The pattern of use of antibiotics in the hospitalized febrile patients was studied and concluded that children with YOS >21 (Table 3) were 
Table 3: Statistical analysis for prediction of bacteremia and clinical outcome by YOS

\begin{tabular}{lllllll}
\hline Parameters against YOS & ROC AUC & Sensitivity (\%) & Specificity (\%) & 1 - specificity & LR+ & LR- \\
\hline Bacteremia (>20) & 0.970 & 100 & 90.2 & 0.098 & 10.2 & 0.00 \\
Outcome (Mortality) $(>21)$ & 0.894 & 90.9 & 85.4 & 0.146 & 6.2 \\
Ventilation support $>21)$ & 0.969 & 100 & 91.7 & 0.083 & 12.04 \\
Antibiotics hike up (>21) & 0.822 & 70.4 & 94.4 & 0.056 & 0.00 \\
\hline
\end{tabular}

YOS: Yale observation scale

Table 4: Correlation of YOS score with clinical outcome parameters

\begin{tabular}{|c|c|c|c|c|c|c|c|}
\hline \multirow[t]{2}{*}{ YOS } & \multicolumn{2}{|c|}{$\begin{array}{l}\text { Requirement of mechanical } \\
\text { ventilation (number of cases) }\end{array}$} & \multicolumn{2}{|c|}{ Final outcome (number of cases) } & \multicolumn{3}{|c|}{$\begin{array}{l}\text { Need for scaling up of antibiotics } \\
\text { (number of cases) }\end{array}$} \\
\hline & Yes & No & Expired & Recovered & More than once & Once & No \\
\hline $6-11$ & 0 & 43 & 0 & 42 & 0 & 3 & 38 \\
\hline $12-17$ & 0 & 29 & 0 & 30 & 1 & 4 & 25 \\
\hline $18-23$ & 4 & 9 & 2 & 11 & 3 & 4 & 6 \\
\hline $24-30$ & 13 & 2 & 9 & 6 & 9 & 3 & 3 \\
\hline
\end{tabular}

Expressed as number of cases with the below parameters against subgroups of YOS showing clustering of cases with good outcome measures (highlighted in green) at lower scores and bad clinical outcome (highlighted in red) at higher scores. YOS: Yale observation scale

Table 5: Other studies on Yale observation scale

\begin{tabular}{|c|c|c|c|c|c|}
\hline \multicolumn{6}{|c|}{ Serious bacterial infections } \\
\hline Feature & Study & $\begin{array}{l}\text { Setting* (prevalence of } \\
\text { serious bacterial infections) }\end{array}$ & Age $^{\#}$ & LR+ $(95 \% \mathrm{CI})$ & LR- (95\% CI) \\
\hline \multirow[t]{6}{*}{ YOS } & McCarthy $1982^{3}$ & Intermediate ${ }^{* *}$ & $<24$ months & $6.7(4-11.1)$ & $0.16(0.13-0.53)$ \\
\hline & Hsiao $2006^{10}$ & Intermediate & 57-180 days & $1.1(0.62-1.98)$ & $0.97(0.82-1.15)$ \\
\hline & Thayyil $2005^{6}$ & Intermediate & $1-36$ months & $2.7(1.72-4.13)$ & $0.19(0.03-1.17)$ \\
\hline & Baker $1990^{8}$ & High & 26-56 days & $2.3(1.32-3.9)$ & $0.68(0.5-0.93)$ \\
\hline & Lacour $2001^{11}$ & High & 7 days - 36 months & $1.6(0.66-3.78)$ & $0.91(0.74-1.12)$ \\
\hline & GalettoLacour $2003^{9}$ & High & 7 days - 36 months & $1.3(0.58-2.92)$ & $0.93(0.74-1.18)$ \\
\hline
\end{tabular}

*The sensitivity and specificity of test changes with change in prevalence of disease under study. ${ }^{* *}$ Categorization on the basis of prevalence of the serious bacterial

illnesses:

- Low prevalence-0-5\%

- Intermediate prevalence-5-20\%

- High Prevalence $\geq 20 \%$

\#Age group included in the study is important as the response to the observation parameters as in YOS varies with the neurological maturity, hence the behavior of a

2 month and 2-year-old child differs; variable age groups included in studies (present study included 3-36 months age). YOS: Yale observation scale

found to require higher antibiotics which signify such patients being infected with possible drug resistant or atypical microbial strains. As the specificity of the test is high, patients presenting to the hospital setting with a YOS $>21$, should be managed with the higher spectrum antibiotics initially and later "downscaling" the same to lower spectrum ones based on blood culture and sensitivity. This result has a lot of clinical relevance as it has been shown by the quoted study [4] that delayed appropriate antibiotics increase mortality and risk of organ dysfunction in bacteremia. As the sensitivity of YOS $>21$ for prediction of need for scaling up antibiotics is low. Hence, YOS $<21$ cannot help to withhold higher antibiotics. These results need further validation in future studies.

Limitations of our study included a small sample size. Further studies on the subject can help in validation of rational clinical approach in daily practice.

\section{CONCLUSION}

- YOS $>20$ is a reliable tool for ruling out bacteremia in a febrile child It is not a good tool for ruling in bacteremia as the scale is affected by many other factors.

- YOS score $>21$ predicts poor prognosis as it signifies more probability of requirement of mechanical ventilation, need for scaling up antibiotics, and increased mortality, hence it differentiates sick and less sick children and impresses upon the requirement of higher antibiotics for YOS $>21$ at admission.
- YOS can be used to monitor admitted patients for early anticipation of deterioration or non-responsiveness to ongoing treatment.

- In view of its simplicity, YOS is a tool which can be used by the skilled and semi-skilled workers to know about the need for referral to higher centers for further management.

\section{REFERENCES}

1. UNICEF-2013 Statistical Snapshot Child Mortality. http://www.who. int/healthinfo/global_burden_disease/ChildCOD_method_2000_2013. pdf.

2. Bang A, Chaturvedi P. Yale observation scale for prediction of bacteremia in febrile children. Indian J Pediatr 2009;76(6):599-604.

3. McCarthy PL, Sharpe MR, Spiesel SZ, Dolan TF, Forsyth BW, DeWitt TG, et al. Observation scales to identify serious illness in febrile children. Pediatrics 1982;70(5):802-9.

4. Weiss SL, Fitzgerald JC, Balamuth F, Alpern ER, Lavelle J, Chilutti M, et al. Delayed antimicrobial therapy increases mortality and organ dysfunction duration in pediatric sepsis. Crit Care Med 2014;42(11):2409-17

5. Thompson M, Van den Bruel A, Verbakel J, Lakhanpaul M, Haj-Hassan T, Stevens R, et al. Systematic review and validation of prediction rules for identifying children with serious infections in emergency departments and urgent-access primary care. Health Technol Assess 2012;16(15):1-99.

6. Thayyil S, Shenoy M, Hamaluba M, Gupta A, Frater J, Verber IG. Is procalcitonin useful in early diagnosis of serious bacterial infections in children? Acta Paediatr 2005;94(2):155-8.

7. Andreola B, Bressan S, Callegaro S, Liverani A, Plebani M, Da Dalt L. 
Procalcitonin and C-reactive protein as diagnostic markers of severe bacterial infections in febrile infants and children in the emergency department. Pediatr Infect Dis J 2007;26(8):672-7.

8. Baker MD, Avner JR, Bell LM. Failure of infant observation scales in detecting serious illness in febrile, 4 - To 8-week-old infants. Pediatrics 1990;85:1040-3.

9. Galetto-Lacour A, Zamora SA, Gervaix A. Bedside procalcitonin and C-reactive protein tests in children with fever without localizing signs of infection seen in a referral center. Pediatrics 2003;112:1054-60.

10. Hsiao AL, Chen L, Baker MD. Incidence and predictors of serious bacterial infections among 57 - To 180-day-old infants. Pediatrics 2006;117(5):1695-701

11. Lacour AG, Gervaix A, Zamora SA, Vadas L, Lombard PR, Dayer JM, et al. Procalcitonin, IL-6, IL-8, IL-1 receptor antagonist and
C-reactive protein as identificators of serious bacterial infections in children with fever without localising signs. Eur J Pediatr 2001;160(2):95-100.

12. Van den Bruel A, Haj-Hassan T, Thompson M, Buntinx F, Mant D. Diagnostic value of clinical features at presentation to identify serious infection in children in developed countries: A systematic review. Lancet 2010;375(9717):834-45.

13. Centers for Disease Control and Prevention. Epidemiology and Prevention of Vaccine-Preventable Diseases. $13^{\text {th }}$ ed. Washington, D.C Centers for Disease Control and Prevention; 2014.

14. Harris JB, Gacic-Dobo M, Eggers R, Brown DW, Sodha SV. Global routine vaccination coverage, 2013. MMWR 2014;63(46):1055-8.

15. UNICEF India 2013 Health Related Statistics Report. Downloadable at; http://www.unicef.org/infobycountry/india_statistics.html. 